\title{
ORIGEN Y FUNDAMENTO DE LA EDUCACIÓN BASADA EN COMPETENCIAS
}

Alejandro López Ibarra*

\section{Resumen}

El presente ensayo trata de establecer cuáles son las premisas históricas y pedagógicas en las que se basa el Modelo Educativo Basado en Competencias. En primer término, se hace una reflexión sobre la situación de la escuela en la Era del Conocimiento y cómo una enseñanza que gire en torno a la promoción de competencias es la que puede dar respuestas a las exigencias del mundo actual. Sin embargo, se hace una distinción entre lo que es una perspectiva estrecha y una perspectiva amplia en la aplicación de este modelo educativo y cómo esta última se presenta como una respuesta plausible para formar a los ciudadanos que requiere esta época tan convulsionada.

\section{Abstract}

The present essay tries to establish which are the historical and pedagogical premises based on the Educative Model Based on Competitions. In first term, a reflection is made on the situation of the school in the Era of Knowledge and how an education that turns around the promotion of competitions is the one that can give answers to the exigencies of the present world. Nevertheless, a distinction is made between a narrow perspective and a wide one in the application of this educative model and how this last one appears like a reasonable answer to train the citizens whom this so convulsed time requires.

PALABRAS CLAVE: Competencias, evaluación del conocimiento, ciudadanía, modelo educativo

*Comunicólogo de profesión, tiene una Especialidad en Estrategias Psicopedagógicas por parte de la Universidad La Salle y está por concluir, en el Tecnológico de Monterrey, sus estudios de la Maestría en Educación con Acentuación en Procesos de Enseñanza y Aprendizaje.

Su carrera docente la inició desde 1997 y ha impartido clases en diversas facultades, pero siempre en asignaturas relacionadas con el Área de las Ciencias Sociales. Ha sido asesor de tesis y sinodal en varios exámenes profesionales.

Actualmente se desempeña como Director de Comunicación, Difusión y Promoción de la Universidad La Salle Cancún donde, a la par de ser el 
responsable de toda la comunicación institucional, está al frente de la Licenciatura en Ciencias de la Comunicación. comunicacion@lasallecancun.edu.mx

La Nueva Educación en la Era del Conocimiento.

Sin lugar a dudas, el mundo se ha transformado a un ritmo desenfrenado en los últimos años. Ya desde el advenimiento de la Revolución Industrial se perfilaban una serie de cambios que impactarían profundamente en el tejido social. Pero lo que hemos experimentado en la llamada Era del Conocimiento es algo para lo que, en muchos sentidos, no estábamos preparados.

Un editorial del Periódico "El Comercio" de Miami (2006, p.1) señalaba sobre la Era del Conocimiento:

Estamos viviendo una transformación radical. Por primera vez, el factor de producción más importante está en manos de los trabajadores y éste es el conocimiento... Las reglas de juego han cambiado. Hemos pasado de la Era Industrial a la Era del Conocimiento. En la sociedad del conocimiento, el trabajador del conocimiento gana acceso al trabajo y posición social a través de la educación. Por lo tanto, la adquisición y distribución de conocimiento formal, tiene la misma importancia que la que ha tenido la adquisición y distribución de la propiedad e ingresos en los últimos siglos.

Por lo tanto, en el pasado, el ser dueño de propiedades y tener un gran capital eran elementos fundamentales para descollar en el terreno profesional. En cambio, en la actualidad, si bien es cierto el tema económico sigue siendo importante, nos encontramos con que el conocimiento es considerado, en sí mismo, un producto valioso y objeto de transacciones comerciales. En este contexto no es de extrañarnos que muchos de los profesionistas más destacados de nuestra época se dediquen a la consultoría. En otras palabras, su conocimiento es el bien que intercambian con otros.

Por ello, al hablar de la Era del Conocimiento, autores como Cardona (2002, p. 2) nos indican que "la educación debe replantear sus objetivos, sus metas, sus pedagogías y sus didácticas si quiere cumplir con su misión en el siglo XXl". Sin embargo, en el entorno nacional e internacional, podemos ver cómo muchas escuelas o universidades no han sabido enfrentarse a las nuevas demandas sociales de nuestra época. Ante esta, llamada por algunos, crisis educativa, no comparto la visión de quienes profetizan el cierre de las escuelas y el surgimiento de un mundo de autodidactas conectados a la Red (Katz, 1999). Empero, estoy plenamente convencido que la escuela del futuro ( $\mathrm{y}$ del presente) necesita cambios estructurales significativos. 
Pero, ¿por qué debemos cambiar a las escuelas? ¿Qué no han hecho bien su trabajo? Es decir, muchas personas podrían argumentar que el gran avance tecnológico y científico que hemos tenido ha sido promovido por individuos que pasaron años preparándose en instituciones educativas con estructuras y métodos tradicionales. Por lo tanto, si los resultados son tan positivos, ¿dónde está el problema? Podríamos analizar si estos científicos y profesionistas han destacado en sus ámbitos de desarrollo, a pesar de las escuelas en las que estudiaron y si su éxito se debe, en gran medida, al autodidactismo. Pero más bien quisiera centrarme en las razones históricas del surgimiento de las universidades y cómo las condiciones imperantes en dicha época han sido rebasadas y, por ende, las instituciones educativas que siguen trabajando bajo el modelo de antaño no están preparadas para responder a las necesidades de un mundo como el actual.

Quisiera enfatizar que este análisis histórico, aunque esté centrado en las universidades, impacta la realidad de todos los niveles educativos; ya que como veremos más adelante, la Educación Basada en Competencias se empezó a implementar en las instituciones de Educación Superior, para luego influir el currículum de escuelas que van desde el preescolar hasta el bachillerato.

Ginés (2004), en su artículo "La necesidad del cambio educativo para la sociedad del conocimiento" comenta que, aunque la universidad como tal surgió en la Edad Media, no fue sino hasta el siglo XIX, en plena Era Moderna, que se fueron configurando los tres modelos universitarios que imperan en muchas de las instituciones de Educación Superior actuales:

a) El Modelo Alemán, cuyo objetivo era que los estudiantes adquirieran una amplia cultura sobre diversos temas, que tuvieran una investigación científica que los sustentara, independientemente de si éstos tenían un uso en el ámbito laboral. La premisa de este modelo era que las personas con un amplio dominio de conocimientos científicos, los aportaran al desarrollo del país en diversas facetas.

b) El Modelo Francés, el cual surgió para responder a las demandas del Imperio Napoleónico cuyo crecimiento demandaba profesionistas en muchos rubros. Por lo tanto, la universidad estaba al servicio de las necesidades del Estado y preparaba a los burócratas que el gobierno necesitaba.

c) El Modelo Anglosajón combinaba algunas de las características de los dos anteriores, pero dándoles ciertos elementos distintivos. Al igual que el Modelo Alemán, pretendía dotar a los estudiantes de un conocimiento amplio sobre diversos temas, pero a diferencia de éste, no promovía la investigación. Asimismo, los estudiantes que concluyeran sus estudios podían terminar trabajando para el Estado, al igual que en el Modelo Francés. Pero lo anterior, no significaba que 
también había una gran posibilidad de que los jóvenes trabajaran para la iniciativa privada. Además, a diferencia de los otros dos modelos, el Modelo Anglosajón fue desarrollado por instituciones privadas y su administración no estaba en manos del Estado.

Poco a poco, estos modelos se fueron combinando; en la actualidad, podemos encontrar en nuestros sistemas universitarios rastros de cada uno. Sin embargo, a pesar de sus diferencias, un rasgo característico de estos tres modelos de universidad era la premisa de que los estudiantes se preparaban para ejercer profesiones que no sufrían grandes cambios con el paso del tiempo. Es decir, era un pensamiento común el hecho de que un egresado de una carrera obtenía una licencia (de ahí, la palabra licenciado) para ejercer una profesión de por vida.

Si nos ubicamos en el siglo XIX y en la importancia que las élites (como las universitarias) le daban a la ciencia exacta, podemos entender que se pretendía transmitir a las nuevas generaciones los principios inamovibles que el genio humano había logrado "arrancarle" a la naturaleza. La enseñanza de leyes era importante, pero no sólo en las Ciencias Naturales. Esa tendencia, también era un anhelo de los estudiosos del mundo social.

Una vez que se ha comprendido el origen de nuestras universidades actuales, nos percatamos cómo sus estructuras y fundamentos han sido rebasados. ¿Por qué basarnos en las necesidades y realidades del Modernismo del siglo XIX cuando nos encontramos ante el influjo del Posmodernismo del siglo XX? ¿Transmitir a unos cuantos, cual tesoro o "iluminación", un conocimiento "eterno" cuando hoy sabemos que hasta ciertos principios que considerábamos inamovibles están sujetos a revisión? El mundo ha cambiado y el conocimiento que adquiere un individuo, cambia cada cinco años (Argudín, 2005). Lejos ha quedado la época en que los egresados de las universidades concluían sus estudios y estaban "preparados para la vida". La Era del Conocimiento nos demanda mucho más que eso y una de las respuestas que se ha generado a esta problemática es la llamada Educación Basada en Competencias.

El movimiento en pro del desarrollo de competencias en los estudiantes universitarios surgió a finales de la década de los 60 y principios de los 70 . En esa época, un profesor de Psicología de Harvard, David McClelland, se percató que los exámenes o pruebas que se aplicaban en las universidades no podían predecir el futuro éxito o fracaso profesional del sustentante (Adams, 1996, citado por Brundrett, 2000). McClelland se empezó a preguntar el porqué ocurría esto y trató de encontrar las variables que le permitirían predecir el futuro profesional de los jóvenes universitarios. Fue en ese momento, que el psicólogo estadounidense fundó la firma consultora "McBer", con el objetivo de encontrar lo que hacía 
competente a un trabajador. En otras palabras, intentaba encontrar los factores o competencias que podían ser determinantes en la adecuada ejecución de una labor, para lo cual elaboró la llamada "Evaluación de Competencia Laboral".

Brundrett (2000) nos narra que en 1981, Richard Boyatzis, un consultor de la empresa "McBer", intentó definir un "Modelo Genérico de Competencia Gerencial". Para ello aplicó la "Evaluación de Competencia Laboral" desarrollada por su jefe a más de 2,000 personas que tenían puestos gerenciales en 12 compañías distintas. La intención de Boyatzis era encontrar las características de un desempeño laboral sobresaliente. Su trabajo derivó en una lista de 19 competencias básicas que todo gerente debía poseer si pretendía realizar su trabajo de forma sobresaliente.

Ahora bien, ¿por qué los resultados del trabajo realizado en la firma "McBer" generaron tantas implicaciones en el terreno educativo? Porque estas investigaciones produjeron una "lista de oro" sobre lo que debía poseer una persona para ser considerada competente en su trabajo. $Y$ aunque el estudio de Boyatzis estaba enfocado a la Administración de Empresas y, particularmente, a la mejora gerencial; las preguntas que empezaban a rondar en las mentes de los educadores de distintas disciplinas eran: ¿será posible generar listas similares de competencias para otras profesiones? Y si esto es posible, ¿por qué no enseñar a las personas esas competencias?

Tratando de poner en contexto la revolución que significó el trabajo de McClelland y Boyatzis, pensemos cómo un maestro "tradicional" enseñaba (o sigue enseñando) las "virtudes del buen gerente" a los alumnos de Administración. Lo más probable es que el maestro les diera textos teóricos sobre el liderazgo que un gerente debe tener sobre su equipo, sobre la importancia de la motivación en el logro de las metas, etc. Es más, quizá podrían hacer discusiones sobre los tópicos y los docentes más "abiertos", incluso, les pedirían hacer simulaciones de los ambientes de trabajo. Pero ¿esas actividades harían que los alumnos, en el momento que egresaran, fueran gerentes competentes? Tristemente, la respuesta es no. Ahora bien, ¿por qué no? Porque la lista de Boyatzis incluía competencias que rara vez son enseñadas en el aula; como el autocontrol o el pensamiento analítico (Carriel, Ruiz, Ruiz y Suazo, 2004). Entonces podemos empezar a vislumbrar el cambio de rumbo que significa la Educación Basada en Competencias. Si nos apegamos al caso que hemos venido explicando, un maestro de Administración que quisiera promover buenos gerentes en "potencia" tendría que diseñar actividades que sometieran al alumno a niveles de stress similares a los que se viven en el ámbito laboral, con el objeto de que el estudiante pudiera ir moldeando su autocontrol. El docente también debería pensar cómo desarrollar en los alumnos la capacidad cognitiva de hacer análisis certeros, pues 
esta competencia es fundamental para que no se pierda el rumbo en la obtención de las metas.

Pero detengámonos un momento a reflexionar sobre lo que tendría que hacer nuestro hipotético maestro. En el párrafo anterior se mencionó que un buen gerente debe tener autocontrol pero, parafraseando nuestro mismo ejemplo, ¿cómo enfrentarse al diseño de actividades que sometan al alumno a niveles de stress similares a los que se viven en el ámbito laboral, con el objeto de que el estudiante pueda ir moldeando su autocontrol? Es más, ¿se puede hacer eso en el aula? Muchos autores piensan que no y que la única manera es llevando la universidad a la empresa. Es decir, el campus deja de estar confinado a las cuatro paredes del salón y los alumnos, vía prácticas profesionales, cátedras empresariales o estancias por proyecto, entran en contacto con la realidad profesional.

Sin embargo, algunas personas pensamos que, si bien es cierto que es muy importante el llevar la universidad a la empresa, también se pueden generar competencias trayendo la empresa a la escuela. El dar conferencias, pláticas o el generar proyectos escolares de manera conjunta con instituciones privadas 0 públicas es otro tipo de acercamiento válido y necesario para promover el desarrollo de alumnos competentes. Además, esta última vertiente no queda circunscrita a la Educación Superior y es una de las formas en que los profesores de otros niveles educativos pueden vincular sus materias con la realidad que sus alumnos enfrentan en su vida cotidiana.

Ahora bien, ¿cómo se definen las competencias? Pensamos que el recorrido que, hasta el momento, hemos tenido sobre el origen y fundamento de este modelo educativo, permite entender algunas de las definiciones que se presentan a continuación. Sin embargo, quisiera hacer hincapié en que éstas son tan variadas como los autores que escriben sobre el tema. Es decir, la Educación Basada en Competencias es un tópico tan nuevo que todavía nos encontramos en un proceso de consenso.

Boyatzis (1982, citado por Brundrett, 2000) definía a las competencias como las características que marcan la diferencia entre una actuación sobresaliente y un desempeño promedio o abajo del promedio. Contrario a lo que los autores estadounidenses como McClelland y Boyatzis sugerían, en el Reino Unido se considera que una persona ha adquirido una competencia cuando puede desempeñar adecuadamente una labor, sin que necesariamente tenga una actuación sobresaliente (Brundett, 2000). Independientemente de esta diferencia, lo que se puede apreciar es que ambas posturas tienen una fuerte tendencia a relacionar sus definiciones con el ámbito empresarial, y pareciera que se confunde 
el término "actuación en el trabajo" con el de competencia. Este hecho es una de las principales críticas a la Educación Basada en Competencias. Muchas voces se alzan en contra de esta dependencia educativa a las necesidades de la empresa y en esa visión tan estrecha que constriñe la capacidad personal con la capacidad laboral.

Hacket (2001) en su artículo "Educando para la Competencia y la Práctica Reflexiva" comenta que, dentro de la Educación Basada en Competencias, se puede considerar que hay dos perspectivas antagónicas entre sí. La perspectiva estrecha, la cual sostiene que el entrenamiento estandarizado produce resultados que pueden ser alcanzados, en un nivel aceptable, por todos los educandos. Los pasos de ese entrenamiento estandarizado surgen de la observación de un trabajador que ha adquirido cierta competencia. En cambio, la perspectiva amplia toma en cuenta las facetas sociales, intelectuales, emocionales y de proceso de las diversas circunstancias educativas en la que la Educación Basada en Competencias es practicada. No confunde el término "actuación en el trabajo" con el de competencia y enfatiza los aspectos humanos en las descripciones sobre competencia.

Considero que el antagonismo entre la perspectiva estrecha y la visión amplia es el punto central de la discusión en torno a la definición de lo que es una competencia; y es en este sentido que, para los fines de este ensayo, se tomará una postura ante esta disyuntiva.

Pienso que la perspectiva estrecha tiene su razón de ser, tanto práctica como histórica. Es decir, los empresarios se percataron que las universidades no estaban "haciendo su trabajo" y los egresados de las mismas llegaban al ámbito laboral sin todos los elementos necesarios para su buen desempeño. Por lo tanto, decidieron generar procesos de capacitación específicos para sus empleados en las áreas en las que consideraban que requerían apoyo. Obviamente, todos estos esfuerzos estaban (y están) encaminados a mejorar la productividad de la empresa y no toman mucho en cuenta a la persona como tal. Sin embargo, el hecho de que exista una justificación del surgimiento de la perspectiva estrecha, no significa que esa deba ser la visión con la que la Educación Basada en Competencias sea llevada al ámbito de las escuelas. Estoy firmemente convencido que la perspectiva amplia, la cual toma en cuenta el desarrollo integral del ser humano, es la que debe prevalecer en los diseños curriculares de las escuelas de todos los niveles. Esta visión más holística no hace a un lado las preocupaciones "pragmáticas y empresariales" de la postura estrecha. Más bien, le añade elementos que son muy valiosos. 
Incluso, la UNESCO (Organización de las Naciones Unidas para la Educación, la Ciencia y la Cultura, por sus siglas en inglés) ha adoptado la perspectiva amplia en su propia definición de lo que es un competencia, la cual es: "conjunto de comportamientos socio afectivos y habilidades cognoscitivas, psicológicas, sensoriales y motoras que permiten llevar a cabo adecuadamente un desempeño, una función, una actividad o una tarea" (Argudín, 2005, p. 12).

Podemos concluir que dependiendo de la perspectiva con la que uno asuma la Educación Basada en Competencias, se podrán determinar los alcances de la aplicación de este modelo educativo en los alumnos. ¿Qué es lo que queremos? ¿Sólo alumnos que sepan cómo hacer sus trabajos y que adquieran competencias procedimentales? Entonces, hagamos uso de la perspectiva estrecha. En cambio, ¿queremos alumnos que sepan hacer su trabajo pero que, al mismo tiempo, tengan una serie de actitudes y comportamientos que los hagan mejores personas y no sólo trabajadores? Si la respuesta es afirmativa, la perspectiva amplia de la Educación Basada en Competencias es lo que estamos buscando. Además, ésta tiene la gran ventaja de adaptarse a todos los niveles educativos y no constreñirse al ámbito universitario.

De todos los textos que abogan por la Educación Basada en Competencias, el escrito por Argudín (2005) parece el más adecuado para expresar los puntos a favor de este modelo educativo. Retomando los puntos más importantes de los primeros tres capítulos del libro de esta autora, podemos señalar que la Educación Basada en Competencias tiene las siguientes ventajas:

a) En primer término, la Educación Basada en Competencias hace frente a una sociedad donde el conocimiento cambia de forma muy rápida. Es decir, si en esta época de transformaciones constantes, el único "tesoro" del estudiante es la información que tiene, la valía de lo que conoce se irá perdiendo con el paso de los años y de una forma acelerada. De lo anterior se deriva que, si el conocimiento se renueva tan rápido, las escuelas tienen que enseñar a los alumnos a aprender a aprender. De esa manera, no importa que la información cambie o sea mucha, el estudiante tendrá la competencia de indagar, sintetizar y valorar los nuevos datos que surjan en su ámbito profesional y personal.

b) También la Educación Basada en Competencias le permite al estudiante identificar el procedimiento utilizado para llevar a cabo las cosas. De esa manera, el alumno podrá tener un autocontrol sobre los pasos que lleva a cabo para lograr las metas que se proponga.

c) La Educación Basada en Competencias evita la desvinculación de los contenidos escolares y las demandas laborales de la sociedad posmoderna. 
d) ¿Cuál es la función de la educación? Que el individuo se integre a la sociedad y que sea transformado bajo las pautas culturales aceptadas. Sin embargo, en el modelo de competencias también se espera que el aprendiz llegue a ocupar un lugar en el sector productivo. Es decir, en una perspectiva amplia de una Educación Basada en Competencias, se pretende generar personas con una buena formación (normas, valores, actitudes, código ético, etc.) y una buena capacitación (conceptos y procedimientos para desempeñar correctamente una función).

\section{FuentesdeConsulta}

ARGUDÍN, Y., (2005), Educación basada en competencias. Distrito Federal, México: Trillas.

BRUNDRETT, M. (2000). The question of competence: the origins, strengths and inadequacies of a leadership training paradigm, School Leadership \& Management Abingdon.

CARDONA, G. (2002). Tendencias educativas para el siglo XXI: educación virtual, on line y @learning. Elementos para la discusión, Revista Electrónica de Tecnología Educativa,

CARRIEL, J., Ruiz, S., Ruiz, N. y Suazo, E. (2004). Diseño de un sistema de evaluación de las competencias a desarrollar por los usuarios de las TIC. (Disertación para obtener el grado de licenciado, Universidad de Concepción en Chile, 2004).

GINÉS Mora, J. (2004). La necesidad del cambio educativo para la sociedad del conocimiento. Revista Iberoamericana de Educación, 35. Disponible en: http://www.campus-oei.org/revista/rie35a01.htm

HACKET, S. (2001), Educating for competency and reflective practice: fostering a conjoint approach in education and training, Journal of Workplace Learning, 13 (3/4).

KATZ, R. (1999), Information Technology and the New Competition in Higher Education. San Francisco, California, USA: Jossey-Bass Higher and Adult Education Series.

Opinión-Hemos pasado de la era industrial a la era del conocimiento; [Fuente: El Comercio]. (28 de Junio). Noticias Financieras, 1. Recuperado en Octubre 22, 2007, de Latin American Newsstand Database. (Document ID: 1068319921). 